\title{
Clinical study on the effectiveness and safety of loose combined cutting seton in the treatment of high perianal abscess: a randomized controlled trial protocol
}

\author{
Yuying Shi ${ }^{1}$, Lihua Zheng ${ }^{1}$, Xin $\mathrm{Li}^{1}$, Congcong Zhi ${ }^{1}$, Yicheng Cheng ${ }^{1}$, Jiaying Shan ${ }^{2}$, Yaxuan Sun ${ }^{2}$, \\ Hongxin Guo ${ }^{2}$, Dun Liu' ${ }^{2}$ Yan Zhang ${ }^{2}$ \\ ${ }^{1}$ Department of Proctology, China-Japan Friendship Hospital, Beijing, China; ${ }^{2}$ Graduate School, Beijing University of Traditional Chinese Medicine, \\ Beijing, China \\ Correspondence to: Lihua Zheng. Department of Proctology, China-Japan Friendship Hospital, Beijing 100029, China. Email: btv1262021@163.com.
}

\begin{abstract}
Background: High perianal abscess is an emergency in the anorectal department. It can result in longterm pain and a huge psychological burden to patients, and seriously affects the quality of life of patients. At present, the effect of antibiotics alone for high perianal abscess is not satisfactory. Loose combined cutting seton (LCCS) can effectively treat high anal fistulas and high perianal abscesses in our clinical practice, but there is no sufficient evidence for its effectiveness in the treatment of high perianal abscesses. The purpose of this study is to observe the effectiveness and safety of LCCS in the treatment of high perianal abscess.

Methods: This study is a single-center, prospective, single-blind, randomized, controlled, non-inferiority clinical study. This study will include patients who are diagnosed with high perianal abscesses and hospitalized for surgery in the Department of Proctology in China-Japan Friendship Hospital (enrollment time: from January 2022 through December 2024). Patients in the experimental group will be treated with LCCS, while patients in the control group will be treated with incision and drainage. Follow-ups will be performed at 1, 3, 7, 14, 21, 28, 90, and 180 days after the operation. The main outcome measures are as follows: (I) cure rate; (II) half-year recurrence rate; (III) postoperative pain visual analog scale (VAS) score; (IV) wound healing time; (V) postoperative anal function evaluation by the Wexner scale; (VI) pressure measurement of the anal canal and rectum before and at half a year after surgery; and (VII) the incidence of adverse events.

Discussion: This study will assess the effectiveness and safety of LCCS in the treatment of high perianal abscess through a strictly designed randomized controlled study, and provides evidence for treatment in clinical practice, thereby improving the treatment effect and improving patients' quality of life.
\end{abstract}

Trial Registration: Chinese Clinical Trial Registry ChiCTR2100049198.

Keywords: Loose combined cutting seton (LCCS); high perianal abscess; effectiveness; safety; protocol

Submitted Oct 29, 2021. Accepted for publication Jan 18, 2022.

doi: $10.21037 /$ atm-22-62

View this article at: https://dx.doi.org/10.21037/atm-22-62

\section{Introduction}

Perianal abscess is an abscess around the anorectum. It is a common clinical emergency in the anorectal department. There are about 2/10,000-10/10,000 new cases each year, and the ratio of males to females can reach 9:1 (1). The main cause of perianal abscess is anal crypt gland infection, proposed by Parks in 1961 (1). Most anorectal abscesses are caused by anal gland infections and blockage of anal gland ducts (1). High perianal abscess refers to the infection space of the abscess which involves the deep space behind the rectum and/or the submucosal space of the rectum and/ or the perianal abscess above the levator ani of the pelvic rectal space abscess (2). Once an infection (especially a deep infection) occurs, it can easily spread to the surrounding 
muscles and connective tissue, and its position is mostly in the intestinal cavity, with a higher distance from the anal margin (3). There is often no obvious fluctuation outside the perianal area, causing prolonged infection and difficulty in treatment, leading to the spread of pus and even serious complications such as sepsis and necrotizing fasciitis.

According to the 2016 version of the American College of Colorectal Surgeons "Guidelines for the Treatment of Perianal Abscess, Anal Fistula, and Rectovaginal Fistula" (4), it is pointed out that incision and drainage are the most important methods for the treatment of perianal abscess. However, it is difficult to apply simple incision and drainage for high perianal abscess in clinical practice. Surgery is the first choice for the clinical treatment of high perianal abscess. The choice of surgical methods has always been a focus in clinical research.

For perianal abscesses, the current domestic common clinical operations include sphincter protection surgery and sphincter injury surgery. Sphincter protection surgery is divided into simple incision and drainage and sphincter preservation surgery. The simple incision and drainage method is the most traditional surgical method for the treatment of perianal abscess. However, for this type of operation, it is difficult to achieve the purpose of radical treatment of high perianal abscess $(5,6)$. Sphincterpreserving surgical methods include intrarectal hanging thread (7) and sphincter-preserving thread hanging (8). According to clinical observations, high perianal abscesses are distorted in the sphincter space, but the scope of inflammation expands and often invades most of the anal straight muscles. This part of the necrotic tissue also needs to be incised and drained, and it is difficult to achieve complete drainage. The disadvantage of sphincterpreserving thread-drawing surgery is that it is difficult to drain the infection beyond the anal straight ring.

The sphincter injury surgical method is commonly used in China. At present, incision and radical resection are mostly used in clinical practice. The more widely used method is incision and drainage radical resection (9). However, the abscess in high perianal abscess exceeds the anal ring, and only the internal orifice is infected with the anal gland and part of the sphincter. For abscesses above the anal ring, it is difficult to enlarge the incision, so it is difficult to perform complete drainage. In order to achieve a more effective treatment of high perianal abscess, we devised an improved surgical method for incision and radical drainage, including low incision + high exclusion (10) and low incision + high thread hanging (11). Incision and exclusion involve cutting the tissue between the infected anal sinus and the incision, as well as part of the internal sphincter and external sphincter, and then a latex tube is used for drainage at a high position. For low incision + high thread hanging, a high position chronic incision and threading are used.

Based on the incision and thread-hanging theory, we perform a solid thread-hanging operation on high perianal abscesses. After the solid thread is disconnected from part of the sphincter, a loose thread is formed. The loose thread is kept for about 21 days to ensure the healing process, and this method is called the loose combined cutting seton (LCCS). This therapy has shown a definite curative effect in the treatment of anal fistula, and the incontinence rate is low and the cure rate is high in the postoperative follow-up visit.

Therefore, in this protocol, we designed a prospective, randomized, controlled, single-blind, and single-center noninferiority clinical trial to compare and observe the clinical efficacy of LCCS in the treatment of high perianal abscesses and the incidence of postoperative complications, in order to provide more evidence when making clinical decisions. Through this study, we will observe the clinical efficacy and safety of LCCS in the treatment of high perianal abscess.

We present the following article in accordance with the SPIRIT reporting checklist (available at https://atm. amegroups.com/article/view/10.21037/atm-22-62/rc).

\section{Methods}

\section{Writing procedure}

The writing of this article is based on the recommendations of the clinical trial protocol and related documents published in the SPIRIT 2013 statement in the "Fournal of Evidence-Based Medicine in Traditional Chinese Medicine" (12).

\section{Study setting}

This study will enroll patients with high perianal abscesses who plan to be admitted to hospital for surgical treatment in the Department of Proctology of the China-Japan Friendship Hospital (Beijing, China).

\section{Enrollment and eligibility criteria}

All patients diagnosed with high perianal abscess (enrollment time: from January 2022 to through December 2024) can participate in this study. The diagnosis of high perianal 
abscesses will be made according to the 2012 Guidelines for the Diagnosis and Treatment of Common Diseases in the Anorectal Department of Traditional Chinese Medicine: perianal throbbing pain or burning pain, fever, white blood cells and neutrophils can be elevated to varying degrees in routine blood tests, digital examination or anal B-ultrasound suggest the abscess is located in the space above the levator ani muscle. With the levator ani muscle as the boundary, high perianal abscess is divided into ischiorectal fossa abscess, retrorectal deep space abscess, and pelvic rectal space abscess. This study was approved by the Chinese Ethics Committee of China-Japan Friendship Hospital (approval number: 2020-89-K53). All procedures performed in this study involving human participants will be in accordance with the Declaration of Helsinki (as revised in 2013). Informed consent will be taken from all the participants.

\section{Inclusion criteria}

Participants must meet all the following criteria to be included in this study: age $\geq 18$ and $\leq 65$ years old, male or female; diagnosed by transrectal B-ultrasound, confirmed to be high perianal abscess; those undergoing anal surgery for the first time; patients will voluntarily participate in clinical trials, provide signed informed consent, and can cooperate with clinical follow-up.

\section{Exclusion criteria}

Potential subjects who meet any of the following criteria will be excluded: those who are unwilling to be followed up; those who have combined high anal fistula during surgery for high perianal abscess; those who have a history of surgery of high perianal abscess; later diagnosed as patients with Crohn's disease, tuberculosis, and other diseases; diabetes, cancer, intestinal tuberculosis, Crohn's disease, AIDS; combined with severe heart, lung, brain, liver, and kidney diseases; women who are pregnant or planning to become pregnant, and women who are breastfeeding.

\section{Intervention and procedures}

Our research process is divided into 3 periods.

The first is the screening period. The main task is to collect cases according to the inclusion and exclusion criteria, and obtain signed informed consent forms before inclusion. Before being selected for the study, the doctor will ask and record medical history and perform screening examinations such as digital rectal examination and intrarectal ultrasound.

The second period is the treatment period, which mainly includes preoperative preparations and surgery. The two groups have the same preoperative preparations. Patients will undergo anorectal cavity color Doppler ultrasound and other examinations before the operation to confirm the diagnosis and confirm that the patient has no contraindications for surgery. Patients will fast for 8 hours before surgery, pass stools normally, and are given an enema if necessary. Surgery plan: For the experimental group (LCCS treatment), patients are placed in a lateral position, routinely disinfected, draped, and given intravenous anesthesia. After the anal canal becomes loose, disinfect the anal canal and perform a digital anal examination to determine the scope and location of the abscess, and then proceed with the abscess. During the operation, an incision is made outside the anus. The incision is selected where the abscess fluctuates the most. After emptying the pus and separating the interval, the probe is inserted from the incision in the abscess cavity into the intestinal cavity. After carefully exploring the inner orifice, the probe will protrude, and infection will be detected. The anal sinus is cut once with the tissue between the incisions and part of the internal sphincter and the external sphincter to remove the necrotic tissue. The index finger explores from the incision and opens the abscess cavity so that the pus is completely removed. First, use the index finger to explore the scope of the abscess cavity, and further separate the separation of the upper abscess cavity, again using middle curved hemostatic forceps to explore the top along the abscess gap. Use the other index finger to extend into the intestinal cavity for guidance, and the tip of the forceps penetrates the intestine at the top of the abscess cavity to form a wall stoma. Withdraw the finger, use 4 10-gauge silk threads, tie one end to the fingertip and put it into the intestinal cavity. Open the hemostatic forceps to clamp this thread, draw the silk thread from the intestinal cavity, gather the two ends of thread, and fix the two ends with a knot. For high horseshoe-shaped perianal abscesses, along the infection area of the abscess cavity, make radial incisions on the same side or both sides of the abscess cavity, and make the opposite mouth with the main incision, and drain the silk thread. Routine cleaning and changing of dressings are performed once a day after the operation. Vaseline gauze is drained and gauze is applied and fixed. About 7 days after the operation, the hanging silk thread 
becomes loose. At this time, the loose thread is drained. After the pus is completely drained, the necrotic tissue detach from normal tissue, and the new granulation tissue is filled, then the thread is removed on the 20th day. For the control group, incision and drainage are used. First, the low incision method is the same as that of LCCS. The probe is probed from the incision of the abscess cavity into the intestinal cavity. The subcutaneous sphincter is incised to remove the necrotic tissue, the index finger explores from the incision, and the abscess cavity is separated and opened so that the pus is completely removed. The index finger moves along the infection gap of the abscess in the intestinal cavity, fully separates the space in the abscess cavity, and places a rubber drainage tube with a side hole at the top of the abscess cavity. Place the upper end of the drainage tube as far as possible at the highest point of the abscess cavity, while the lower end of the rubber tube is sutured along the abscess cavity and fixed to the incision of the anal margin. The drainage tube should be routinely flushed when changing the dressing after the operation. Postoperative medication and nursing: both groups of patients will receive routine intravenous infusion of broadspectrum and anti-anaerobic antibiotics for 3 days after surgery. Those who have bowel movements within 24 hours after the operation should change the dressing immediately after the bowel movement. For those who have a bowel movement more than 24 hours later, the dressing will be changed 24 hours after the operation. Before the dressing change, the traditional Chinese medicine preparations in our hospital are used to fumigate and wash the sitz bath for 10 minutes, and the dressings of the two groups are changed once a day. The prescription for in-hospital preparations for anorectal fumigation and washing is $15 \mathrm{~g}$ of gallnut $15 \mathrm{~g}, 30 \mathrm{~g}$ of dandelion, $15 \mathrm{~g}$ of raw Platycladus orientalis, $30 \mathrm{~g}$ of Sophora flavescens, $15 \mathrm{~g}$ of Glauber's salt, $15 \mathrm{~g}$ of atractylodes, $15 \mathrm{~g}$ of Radix sylvestris, $15 \mathrm{~g}$ of fangfeng, 30 of phellodendron, 15 of red peony root, $15 \mathrm{~g}$ of honeysuckle, and $10 \mathrm{~g}$ of raw licorice. Usage: wrap these with gauze, put it in a basin, and brew with $2,000 \mathrm{~mL}$ hot water. When the water temperature is about $40{ }^{\circ} \mathrm{C}$, wash the anus and wound for 10 minutes twice a day.

The third period is the follow-up period. Follow-up plan: the follow-up time is half a year, including 8 medical visits. Telephone follow-up or face-to-face follow-up will be conducted on the first consultation day and at 1, 3, 7, 14, 21, 28,90 , and 180 days after surgery. The main content of the follow-up includes the patient's current clinical symptoms.

Since the longest postoperative recovery period is about 3 months, we will perform anorectal ultrasound, rectal pressure measurement, anal function score, and anal Wexner incontinence score evaluation on patients before and at 3 months after surgery. At other follow-up, we will mainly focus on the patient's self-reported symptoms and recurrence. If necessary, we will reassess the patient's anal function at the 6-month follow-up and 1-year follow-up. This is because we found through clinical observation that some patients may experience transient anal incontinence due to the effect of postoperative scars. Therefore, the addition of anal function assessment can better evaluate the patient's postoperative anal recovery. A summary of the contents of the research phase is shown in Table 1.

\section{Outcome measures}

\section{Cure rate}

The clinical efficacy evaluation is based on the "Diagnostic Efficacy Criteria for Anorectal Diseases in Traditional Chinese Medicine" devised by the State Administration of Traditional Chinese Medicine. Cure refers to symptoms and signs disappear, and wounds are healed (wound healing rate is $100 \%$ ); improvement refers to symptoms and signs are improved, $50 \% \leq$ wound healing rate $<100 \%$; unhealed refers to there is no change in symptoms and signs (wound healing rate $<50 \%$ ).

\section{Half-year recurrence rate}

The recurrence rate of high perianal abscesses is calculated from the initial calculation of the patient's enrollment, and the patient's high perianal abscess is followed up after half a year.

\section{Postoperative visual analog scale (VAS) scoring}

Record the patient's pain on the 1st, 3rd, 7th, 14th, 20th, and 28th day after suture removal, and use the VAS scoring system. To do this, take a standard horizontal ruler with a length of $10 \mathrm{~cm}$, and the horizontal ruler scale indicates different pain levels. Ask the patient to draw a mark on the horizontal line based on how they feel, and convert it into a score. The pain classification is as follows: 0 points: no pain; 1-4 points: slight pain, the patient can tolerate it, no need to manage it; 5-7 points: the patient has pain and affects sleep, which can be relieved by oral general analgesics; $8-10$ points: the patient has intense pain, the pain is unbearable, they need drugs such as meperidine to relieve it.

Wound healing time: record the time when the wound is completely healed and epithelialization is complete after the 
Table 1 Schedule of subjects

\begin{tabular}{|c|c|c|c|c|c|c|c|c|c|c|c|c|c|}
\hline \multirow{3}{*}{ Time } & \multicolumn{13}{|c|}{ Research stage } \\
\hline & \multirow{2}{*}{$\begin{array}{c}\text { Enrollment } \\
-2\end{array}$} & \multirow{2}{*}{$\begin{array}{c}\text { Randomization } \\
-2\end{array}$} & \multirow{2}{*}{$\begin{array}{r}\text { BS } \\
-1\end{array}$} & \multirow{2}{*}{$\frac{\text { IS }}{0}$} & \multicolumn{8}{|c|}{ PS } & \multirow[t]{2}{*}{ Outcome } \\
\hline & & & & & 1 & 3 & 7 & 14 & 21 & 28 & 90 & 180 & \\
\hline \multicolumn{14}{|l|}{ Enrollment } \\
\hline Consent & $\bullet$ & & & & & & & & & & & & \\
\hline Randomization & & - & & & & & & & & & & & \\
\hline \multicolumn{14}{|l|}{ Intervention } \\
\hline LCCS & & & & - & & & & & & & & & \\
\hline Presentation & & & • & & - & - & - & - & - & - & $\bullet$ & • & \\
\hline Ultrasound & & & - & & & & & & & & 0 & & \\
\hline Pressure & & & $\bullet$ & & & & & & & & $\bullet$ & & \\
\hline VAS score & & & $\bullet$ & & - & - & - & $\bullet$ & - & - & • & • & \\
\hline Wexner score & & & - & & & & & & & & $\bullet$ & $\bullet$ & \\
\hline Anal function & & & & & & & & & & & $\bullet$ & $\bullet$ & \\
\hline Adverse events & & & & & $\bullet$ & - & $\bullet$ & $\bullet$ & $\bullet$ & $\bullet$ & $\bullet$ & $\bullet$ & \\
\hline Healing time & & & & & & & & & - & - & $\bullet$ & - & \\
\hline
\end{tabular}

- represents items should be done at that time point. BS, before surgery; IS, intro-surgery; PS, post-surgery; LCCS, loose combined cutting seton; VAS, visual analog scale.

operation.

\section{Wexner incontinence score}

Measure the Wexner incontinence score before and at half a year after surgery (Table 2).

\section{Measurement of anorectal pressure}

Measure the resting pressure of the anorectal canal, the length of the anal canal high pressure area, and the maximum systolic pressure of the anal canal before and at half a year after the operation. The patient is required to return for this examination after the wound is completely healed.

Incidence rate of adverse events: observe and record the occurrence of adverse events such as urinary retention, anal edema, wound infection, anal incontinence, and anal canal defects in patients after the operation.

\section{Primary outcomes}

The main efficacy evaluation indicators are as follows: (I) postoperative wound and fistula complete cure rate; (II) half-year recurrence rate. Collect data, compare the differences between the two groups in terms of recovery rate, marked effectiveness rate, inefficiency, average healing time, and recurrence rate, and use anorectal B-ultrasound as an objective evaluation index to evaluate the effectiveness of LCCS.

\section{Secondary outcomes}

The secondary efficacy evaluation indicators are as follows: (I) VAS score for postoperative pain; (II) measurement of anal and rectal pressure before and after treatment; (III) Wexner score for anal function before and after treatment; 
Table 2 Wexner incontinence score

\begin{tabular}{|c|c|c|c|c|c|}
\hline Incontinence & \multicolumn{5}{|c|}{ Frequency } \\
\hline Solid & 0 & 1 & 2 & 3 & 4 \\
\hline Fluid & 0 & 1 & 2 & 3 & 4 \\
\hline Gas & 0 & 1 & 2 & 3 & 4 \\
\hline Lifestyle changes & 0 & 1 & 2 & 3 & 4 \\
\hline
\end{tabular}

$0=$ normal; $20=$ complete incontinence; seldom $=\leq 1 /$ month; sometimes $=\leq 1 /$ week,$\geq 1 /$ month; often $=\leq 1 /$ day, $\geq 1 /$ week; always $=\geq 1 /$ day .

(IV) incidence of adverse events.

\section{Sample size}

This study is a non-inferiority clinical randomized controlled trial. The experimental group is treated with LCCS, and the control group is treated with incision and drainage. The cure rate is the main outcome indicator. $\mathrm{N}$ is the sample size. The ratio of the experimental group and the control group is 1:1. According to previous literature and clinical application, the healing rate of control group and the experimental group was set to be $95 \%$. P2 of the control group was set at 0.95 . $\alpha$ is the inspection level, with $\alpha=0.025$ (one-sided) and power $=0.80$. Based on clinical experience, the research team set the non-inferiority threshold as $\mathrm{d}=0.15$. Using PASS15, a sample size of $\mathrm{N} 1=34$ for the experimental group was obtained, and the sample size of the control group is N2=34 cases. Assuming that the loss to follow-up rate of the research subjects is $10 \%$, the sample size is $\mathrm{N} 1=34 / 0.9=38$ cases and $\mathrm{N} 2=34 / 0.9=38$ cases.

\section{Recruitment}

Recruitment for this trial will be carried out through the internet, public account release of the subject recruitment information, and outpatient posting of subject recruitment. After the subjects sign up, they will become enrolled subjects after passing through the regular medical treatment process and screening by the inclusion and exclusion criteria.

\section{Method of allocation of interventions}

\section{Randomization and blinding}

Before joining, we will tell the patients that during the treatment process, the surgeon will use a method with a definite curative effect to perform the operation, which may be a traditional method or a relatively novel method. In order to minimize bias in the trial, after the clinical follow-up is completed, the data will be sent to a third party for analysis and statistics. The third party does not know whether the data belongs to the experimental group or the control group before the results are revealed. Finally, the physician and the statistical analysis party jointly reveal the corresponding grouping of the results.

Random method: the assigned serial number is generated by the anorectal doctor of China-Japan Friendship Hospital using SAS software. According to the random number table generated by SAS software, the random sequence is formed and divided into two groups according to the random sequence number. The larger number is the A group, and $\mathrm{A}$ is the experimental group. The group with the smaller number is group B, and group B is the control group who will be treated by incision and draining. Then, the two sets of random sequences were placed in sealed opaque envelopes and arranged into a random sequence. The envelopes are kept by an appointed investigator. At the time of allocation, the selected cases will receive random serial numbers and corresponding treatment methods according to the time sequence of admission to the hospital, and follow-up treatment will be carried out in accordance with the treatment methods.

\section{Data collection}

\section{Statistical analysis}

The SPSS 22.0 statistical software package was used for statistical analysis. All statistical tests were performed using two-sided tests, and a $\mathrm{P}$ value of $<0.05$ would be considered statistically significant. 
The Kaplan-Meier method was used to estimate the healing rate at each time point after surgery, and the log rank test method was used to compare the healing time between the two groups. The mean \pm standard deviation was used to describe the resting pressure of the anorectal canal, the length of the anal canal high pressure area, and the maximum systolic pressure of the anal canal before treatment and after healing in the experimental group and the control group. The above indicators before treatment were used as covariates, and analysis of covariance was used to compare whether the transformation of the above indicators between the test group and the control group was significantly different after surgery. Outpatient follow-up, telephone follow-up, email, WeChat, and other follow-up methods were used to ensure the completeness and accuracy of follow-up. The data is managed by a dedicated person and checked by 2 persons.

Before comparison of quantitative data between two groups, for example, when comparing the final healing times of wounds and fistulas between the experimental group and the control group, normality and homogeneity of variance should be considered. If normality and uniform variance are met, the t-test will be used, t' test will be used when uneven variance exist. The Wilcoxon rank sum test is used for non-normal data. For comparisons of qualitative data between the two groups, such as the results of anal function curative effect evaluation, the $\chi^{2}$ test is used. Categorical data used the rank sum test. The paired signedrank test is used to compare the changes of incontinence scores before and after treatment in each group, and the one-way ordered Cochran-Mantel-Haenszel $\chi^{2}$ test is used to compare between groups.

\section{Adverse events}

We will record adverse events during the whole study period. Clinical adverse events may occur during treatment. Once adverse events occur, the time of occurrence, clinical manifestations, treatment process and duration, outcome, and the relationship between the drug and adverse events and related events should be recorded in detail on the case report form. If there is an abnormal laboratory test, the patient must be followed up until the test result returns to normal or to the level before medication, otherwise it is determined as having nothing to do with the medication. Serious adverse events should be recorded on the serious adverse event form and reported to the ethics committee within 24 hours.

\section{Ethics committee review}

For this protocol, written informed consent and request for materials directly related to the subjects must be submitted to the ethics committee, and the research can be formally carried out after obtaining the written approval of the ethics committee. The investigator must submit a research report to the ethics committee at least annually (if applicable). When the study is suspended and/or completed, the investigator must notify the ethics committee in writing. The investigator must promptly report to the ethics committee all changes in the research work (such as the revision of the protocol and/or the number of informed consents). These changes may not be implemented until approved by the committee, unless they are made to eliminate obvious and direct risks to the subject. In such cases, the ethics committee will be notified.

\section{Informed consent}

\section{Procedure for obtaining informed consent}

The researcher must provide the subject or their legal representative with an easy-to-understand informed consent form approved by the ethics committee, and give the subject or their legal representative sufficient time to consider this research. Subjects are not allowed to join the study before obtaining the signed written informed consent. During the subject's participation, all updated informed consent forms and written information will be provided to the participants. The informed consent form should be kept as an important document for clinical trials for future reference.

\section{Confidentiality measures}

The results of the research through this project may be published in medical journals, but we will keep the patient's information confidential in accordance with the requirements of the law, and unless required by relevant laws, the patient's personal information will not be disclosed. When necessary, government management departments, hospital ethics committees, and their related personnel can consult the patient's data in accordance with regulations.

\section{Discussion}

Because the anatomical relationship between the anal canal 
and the surrounding tissues of the rectum is complicated, once infection (especially deep infection) occurs, it can easily to spread to the surrounding muscles and connective tissues. Its position is mostly in the intestinal cavity, higher from the anal margin and outside the perianal tissue. There is often no obvious fluctuation, causing the infection to persist and remain unhealed, leading to the spread of pus, and even serious complications such as sepsis and necrotizing fasciitis. This induces long-term pain and brings a huge psychological burden to the patient, and seriously affects the patient's quality of life.

Studies have shown that the effect of antibiotic treatment for high perianal abscesses is not satisfactory. Surgery is the first choice for the clinical treatment of high perianal abscess. The anorectal ring is the control center of the anus. It is composed of the internal and external sphincter, puborectalis, levator ani muscle, and other muscles. It has important functions in maintaining anal control and assisting defecation. The high perianal abscess cavity invades and exceeds the anal straight ring. If it is completely incised and drained, there will be damage to anal function (especially the levator ani muscle and anal sphincter) (13), resulting in anal incontinence. Protecting the anal muscles and using only incision and drainage may lead to poor drainage of the infection site in the high space, effusion and pus, and the inability to completely drain the lesion, resulting in a high recurrence rate (about 44\%) (14) and the occurrence of anal fistula. Therefore, high perianal abscess belongs to the category of refractory diseases in proctology, and its pain degree, high recurrence rate, and postoperative anal incontinence can cause severe psychological and financial burden to patients.

How to improve the success rate of high perianal abscess treatment, reduce recurrence, avoid anal function damage, reduce pain, and improve the quality of life of patients have always been difficult problems in clinical practice. For the treatment of high perianal abscesses, there is still no unified standard at home and abroad. Therefore, exploring suitable treatment methods, focusing on the protection of anal function, reducing recurrence, and improving the quality of life of patients with high perianal abscesses have been pursued by anorectal specialists. Regarding the surgical treatment of high perianal abscesses, simple incision and drainage is the most traditional surgical method for the treatment of perianal abscesses. It is difficult to achieve complete drainage and cure of abscesses of the high straight anal ring and above after anal margin incision alone. Therefore, doctors will also adopt a staged surgery plan, involving one-stage incision and drainage and a two-stage radical surgery plan. Sphincter protection surgery includes intrarectal thread hanging and retaining sphincter thread hanging. Although the sphincter will not be damaged during the operation, it is difficult to drain the necrotic cavity and infected necrotic tissue at the level of the anal straight ring. Furthermore, while simple incision and radical surgery and incision and exclusion cut the tissue between the infected anal sinus and the incision, as well as part of the internal sphincter and external sphincter, it is still difficult to achieve a complete radical cure for the treatment of the high perianal abscess.

At present, incision and thread-hanging surgery is the mainstream surgical procedure in China. The low incision + high thread hanging operation involves cutting the anal canal and rectal ring with a rubber band to tighten the ends of the anal canal and gradually tighten the line after the internal opening and the infected anal sinus are cut to gradually cut off the anal straight ring. Although this kind of operation can be performed slowly without cutting off the infected straight anal ring, it can better protect the integrity of the anorectal ring. However, most scholars currently believe that the incision and thread-hanging operation will still cause some damage to the anal tissue, leaving scars after the operation, and partial anal incontinence. In order to reduce the occurrence of adverse reactions after the threadhanging operation, there are also some improved threadhanging procedures in clinical practice, including highposition virtual thread-hanging, high-position shallow thread-drawing, and other surgical procedures. However, in clinical practice, anal incontinence such as fluid leakage cannot be avoided.

LCCS is an improved new surgical method for the treatment of high perianal abscesses (15). Based on the incision and thread-hanging theory, we perform LCCS on high perianal abscesses. Firstly, the "solid thread" during the operation is used to ligate and thread the abscess cavity or infection space above the internal orifice during the operation. After part of the sphincter is disconnected, a virtual thread is formed, and the silk thread is loosened to ensure drainage during the healing process. The function of patency is to remove the suture after the abscess cavity is drained clean and the granulation tissue is filled, so that the straight anus ring will not be broken. This innovative method is called LCCS, and with all respect, we would like call it Zheng's technology since Zheng et al. established this strategy (15).

This therapy has shown definite curative effect in the 
treatment of high anal fistula and perianal abscess, with a low incontinence rate and high cure rate at the postoperative follow-up visit. The surgical method and follow-up we proposed here have been supported by data (15). Therefore, we will perform a single-center, prospective research trial to observe the clinical efficacy of Zheng's technology in the treatment of high perianal abscesses and the incidence of postoperative complications, and further verify the effectiveness and safety of Zheng's technology, also named LCCS.

\section{Acknowledgments}

Funding: None.

\section{Footnote}

Reporting Checklist: The authors have completed the SPIRIT reporting checklist. Available at https://atm.amegroups. com/article/view/10.21037/atm-22-62/rc

Conflicts of Interest: All authors have completed the ICMJE uniform disclosure form (available at https://atm. amegroups.com/article/view/10.21037/atm-22-62/coif). The authors have no conflicts of interest to declare.

Ethical Statement: The authors are accountable for all aspects of the work in ensuring that questions related to the accuracy or integrity of any part of the work are appropriately investigated and resolved. This study was approved by the Chinese Ethics Committee of China-Japan Friendship Hospital (approval number: 2020-89-K53). All procedures performed in this study involving human participants will be in accordance with the Declaration of Helsinki (as revised in 2013). Informed consent will be taken from all the participants.

Open Access Statement: This is an Open Access article distributed in accordance with the Creative Commons Attribution-NonCommercial-NoDerivs 4.0 International License (CC BY-NC-ND 4.0), which permits the noncommercial replication and distribution of the article with the strict proviso that no changes or edits are made and the original work is properly cited (including links to both the formal publication through the relevant DOI and the license). See: https://creativecommons.org/ licenses/by-nc-nd/4.0/.

\section{References}

1. Shi RJ. A review of new advances in the diagnosis and treatment of anorectal abscess. Chin J Integr Trad Chin Western Med 2018;4:399-41.

2. Chinese Society of Traditional Chinese Medicine. Guidelines for the diagnosis and treatment of common diseases in the Anorectal Department of Traditional Chinese Medicine. Beijing: China Press of Traditional Chinese Medicine 2012:10-6.

3. Liu YR. The clinical value of high-frequency color Doppler ultrasound in the diagnosis of perianal abscess and anal fistula. Chin J Anore Dis 2012;32:12-3.

4. American Association of Colorectal Surgeons. 2011 edition of the American perianal abscess and anal fistula treatment guidelines. Chin J Gastro Surg 2012;15:640-3.

5. Macfie J, Harvey J. The treatment of acute superficial abscesses: a prospective clinical trial. Br J Surg 1977;64:264-6.

6. Malik AI, Nelson RL, Tou S. Incision and drainage of perianal abscess with or without treatment of anal fistula. Cochrane Database Syst Rev 2010;(7):CD006827.

7. Xu ZP, Wang Y, Wang Y. Therapeutic mechanism of wall-hanging suture in the rectum. Chin J Anore Dis 1990;10:12-3.

8. Gu YF. The clinical application of thread-drawing therapy in the treatment of perianal abscess. Jiangsu Tradl Chin Med 2006;27:7-8.

9. Liu Z, Liu LJ, Zhang L. One-time incision and radical resection for the treatment of 198 cases of perianal abscess. West Med 2008;20:637.

10. Lin H, Sun J. Research progress in the application of traditional Chinese medicine absent treatment in the operation of horseshoe-type perianal abscess. J Liaoning Univ Trad Chin Med 2013;2:119-21.

11. Sun J, Peng BK. One-stage incision and thread-drawing in the treatment of perianal abscess in the rectum and anal canal. Chin Commu Phys 2007;9:55.

12. Zhong LD, Zheng SH, Wu TX, et al. SPIRIT 2013 Statement: Defining Standard Items for Clinical Research Programs. Chin J Evid-Based Med 2013;12:1501-7.

13. Yano T, Asano M, Matsuda Y, et al. Prognostic factors for recurrence following the initial drainage of an anorectal abscess. Int J Colorectal Dis 2010;25:1495-8.

14. Wang Y, Tian GB, Zhang R, et al. Prevalence, risk factors, outcomes, and molecular epidemiology of mcr-1-positive Enterobacteriaceae in patients and healthy adults from 
China: an epidemiological and clinical study. Lancet Infect Dis 2017;17:390-9.

15. Zhi CC, Shi YY, Cui WH, et al. A clinical study on the treatment of retrorectal deep space abscess with combination of deficiency and excess, thread-drawing method. Chin J Clin 2019;47:956-8.

(English Language Editor: C. Betlazar-Maseh)

Cite this article as: Shi Y, Zheng L, Li X, Zhi C, Cheng Y, Shan J, Sun Y, Guo H, Liu D, Zhang Y. Clinical study on the effectiveness and safety of loose combined cutting seton in the treatment of high perianal abscess: a randomized controlled trial protocol. Ann Transl Med 2022;10(2):108. doi: 10.21037/ atm-22-62 